
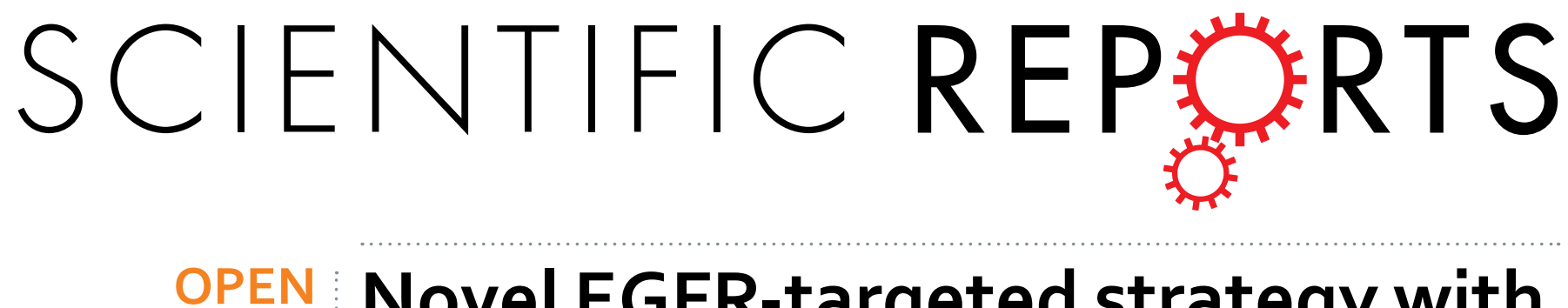

\title{
Novel EGFR-targeted strategy with hybrid peptide against oesophageal squamous cell carcinoma
}

Received: 05 October 2015

Accepted: 10 February 2016

Published: 09 March 2016

\section{Osamu Kikuchi ${ }^{1}$, Shinya Ohashi², Tomohisa Horibe ${ }^{3}$, Masayuki Kohno ${ }^{3}$, Yukie Nakai ${ }^{2}$ Shin'ichi Miyamoto ${ }^{1}$, Tsutomu Chiba ${ }^{1}$, Manabu Muto ${ }^{2} \&$ Koji Kawakami ${ }^{3}$}

Epidermal growth factor receptor (EGFR) is a key molecule in the pathophysiology of oesophageal squamous cell carcinoma (OSCC). However, EGFR-targeted agents such as anti-EGFR antibody or tyrosine kinase inhibitors for OSCC have not demonstrated any clinical benefits. Recently, a novel chemotherapeutic agent, EGFR(2R)-lytic hybrid peptide, a composite of EGFR-binding peptide and lytic peptide fragments, has been shown to exhibit a potent anti-tumour effect against cancers that express high EGFR levels. In this study, we investigated the validity of employing EGFR(2R)-lytic hybrid peptide against OSCC cells both in vitro and in vivo. Additionally, the toxicity of this peptide was assessed in mice. We found high EGFR expression levels on the cell surface of OSCC cells, and the EGFR-binding peptide fragment showed high affinity for OSCC cells. A potent cytotoxic effect was induced within 30 minutes by the exposure of OSCC cells to EGFR(2R)-lytic hybrid peptide. Furthermore, EGFR(2R)-lytic hybrid peptide markedly suppressed the tumour growth of OSCC cells in a xenograft model. Moreover, it did not cause any identifiable adverse effects in mice. Taken together, EGFR(2R)-lytic hybrid peptide was shown to be a valid therapeutic agent against OSCC, providing a crucial rationale regarding novel EGFR-targeted therapies against OSCC.

Oesophageal squamous cell carcinoma (OSCC) is a major histologic type of oesophageal cancer ${ }^{1}$, and the key therapy against advanced or metastatic OSCC is chemotherapy ${ }^{2-4}$. However, the therapeutic effect of anti-cancer agents that can be administered to treat OSCC is still limited. Indeed, combination chemotherapy with 5-fluorouracil (5-FU) and a platinum-based drug for metastatic OSCC patients has been reported to lead to response rates of $30-40 \%{ }^{5-7}$. In addition, docetaxel induced a partial response in only $20 \%$ of OSCC patients ${ }^{8}$.

Epidermal growth factor receptor (EGFR) is frequently (71-88\%) expressed in OSCC tissues ${ }^{9}$, and its expression is associated with a poor outcome ${ }^{10}$. Several clinical trials of EGFR-targeted therapies, such as anti-EGFR antibodies and EGFR tyrosine kinase inhibitors (TKI), have been conducted for patients with advanced OSCC; however, the blockade of EGFR signalling has not been shown to sufficiently improve the outcome of those patients ${ }^{11-14}$. These data suggest that another strategy is required to facilitate the development of EGFR-targeted therapies against OSCC.

Recently, an EGFR-lytic hybrid peptide was developed as a new agent of EGFR-targeted therapy ${ }^{15}$. This peptide consists of two unique components: an EGFR-binding peptide fragment and a lytic peptide fragment (Fig. 1A). The EGFR-binding peptide fragment shows high-level affinity for human EGFR on the cell surface, whereas the lytic peptide fragment exhibits an amphipathic helical structure due to the presence of lysines $(\mathrm{K})$ and leucines (L), and penetrates cellular membranes ${ }^{15}$. Thus, EGFR-lytic hybrid peptide could kill EGFR-expressing cells through the combined process of specific binding to EGFR on the cell surface and subsequently disintegrating cell membranes ${ }^{15}$.

A modified version of EGFR-lytic hybrid peptide has since been developed to improve its binding ability to EGFR by changing the second amino acid of the EGFR-binding sequence from histidine $(H)$ to arginine $(R)^{16}$, and this was designated EGFR(2R)-lytic hybrid peptide (Fig. 1B). The specific binding ability of EGFR(2R)-lytic hybrid peptide to recombinant EGFR was revealed by a binding assay and biophysical analysis using surface

${ }^{1}$ Department of Gastroenterology and Hepatology, Kyoto University Graduate School of Medicine, Kyoto 606-8507, Japan. ${ }^{2}$ Department of Therapeutic Oncology, Kyoto University Graduate School of Medicine, Kyoto 606-8507, Japan. ${ }^{3}$ Department of Pharmacoepidemiology, Graduate School of Medicine and Public Health, Kyoto University, Kyoto 606-8501, Japan. Correspondence and requests for materials should be addressed to K.K. (email: kawakami. koji.4e@kyoto-u.ac.jp) 
(A)

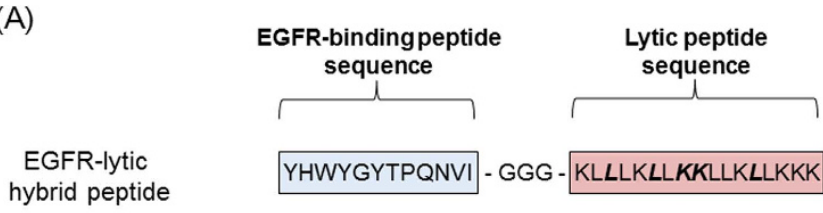

(B)
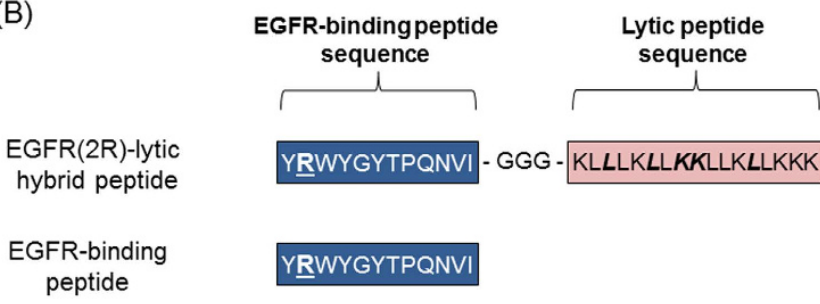

YRWYGYTPQNVI - GGG - KLLLKLLKKLLKLLKKK

YRWYGYTPQNVI

Lytic peptide

KLLLKLLKKLLKLLKKK

Figure 1. Scheme of peptides. (A) Original EGFR-lytic hybrid peptide consisting of an EGFR-binding peptide fragment and a lytic peptide fragment, with three glycine (G) spacers. (B) EGFR(2R)-lytic hybrid peptide consisting of a modified EGFR-binding peptide fragment, three glycine spacers, and a lytic peptide fragment. "(2R)" means that the second amino acid of the EGFR-binding peptide fragment is arginine (R, bold and underlined). Bold and italic letters are D-amino acids, whereas normal letters are L-amino acids. Sequences of EGFR-binding peptide and lytic peptide fragments are also shown.

plasmon resonance experiments ${ }^{16,17}$, and cytotoxic and anti-tumour effects of EGFR(2R)-lytic hybrid peptide were shown to be stronger than those of the original EGFR-lytic hybrid peptide ${ }^{16}$.

Based on these findings, we addressed the validity of EGFR(2R)-lytic hybrid peptide as a novel EGFR-targeted therapy against OSCC.

\section{Results}

EGFR expression and affinity of EGFR-binding peptide for surface of OSCC cells in vitro. Firstly, we examined the expression of EGFR in whole-cell lysates of OSCC cells by Western blotting. As shown in Fig. 2A, EGFR was highly expressed in all OSCC cells, while HEK293 cells, human embryonic kidney cells, showed low-level EGFR expression. Accordingly, we used HEK293 cells as negative control cells. Next, we investigated EGFR expression on the surface of OSCC cells by flow cytometry. As with the results of EGFR expression in whole-cell lysates, EGFR was expressed on the surface of all OSCC cells, but not HEK293 cells (Fig. 2B).

Then, we examined the affinity of EGFR-binding peptide for OSCC cells (TE-11R cells) and HEK293 cells. Although EGFR-binding peptide minimally bound to HEK293 cells, the affinity of EGFR-binding peptide for TE-11R cells was significantly increased in time- (Fig. 2C) and dose- (Fig. 2D) dependent manners.

Cytotoxicity of EGFR(2R)-lytic hybrid peptide against OSCC cells in vitro. Cytotoxic effects of EGFR(2R)-lytic hybrid peptide, the EGFR-binding peptide fragment, lytic peptide fragment, and the co-administration of EGFR-binding peptide and lytic peptide fragments on OSCC cells were investigated. As shown in Fig. 3, EGFR(2R)-lytic hybrid peptide showed a potent cytotoxicity against all OSCC cells, whereas the cytotoxic effects of the EGFR-binding peptide fragment, lytic peptide fragment, and co-administration of EGFR-binding peptide and lytic peptide fragments were limited. The $\mathrm{IC}_{50}$ values of EGFR(2R)-lytic hybrid peptide for each OSCC cell are shown in Supplementary Table S1.

Rapid disintegration of OSCC cell membranes after exposure to EGFR(2R)-lytic hybrid peptide in vitro. We examined the morphologic change of OSCC cells (TE-11R cells) after exposure to EGFR(2R)-lytic hybrid peptide. As shown in Fig. 4A, protrusion from cell membranes was observed within 5 minutes after EGFR(2R)-lytic hybrid peptide exposure. Consistently, levels of extracellular LDH, a marker of cell membrane damage, were increased in the culture medium on exposure to EGFR(2R)-lytic hybrid peptide in a time-dependent manner (Fig. 4B).

Furthermore, to assess the real-time cytotoxicity in cells treated with this peptide, we conducted the ATP bioluminescence assay. Figure $4 \mathrm{C}$ shows that the ATP level was reduced to near zero within 30 minutes following exposure to EGFR(2R)-lytic hybrid peptide (Fig. 4C, Supplementary Fig. S1).

Anti-tumour effect of EGFR(2R)-lytic hybrid peptide against xenografted OSCC tumours. To investigate the anti-tumour effect of EGFR(2R)-lytic hybrid peptide against OSCC cells in vivo, xenografted tumours generated from TE-11R cells were treated with EGFR(2R)-lytic hybrid peptide. The EGFR(2R)-lytic hybrid peptide significantly suppressed the tumour growth of TE-11R cells (Fig. 5A) and induced tumour cell death (Fig. 5B), whereas the lytic peptide fragment without the EGFR-binding fragment did not show anti-tumour effects. 
(A)

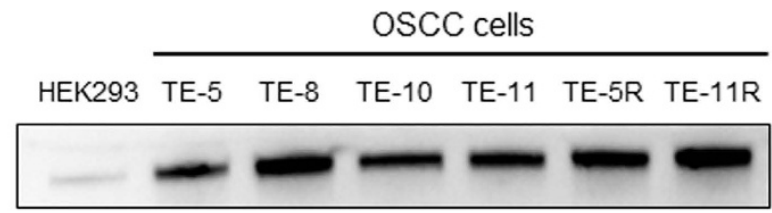

$\beta$-Actin

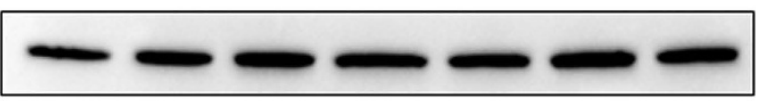

(B)

HEK293

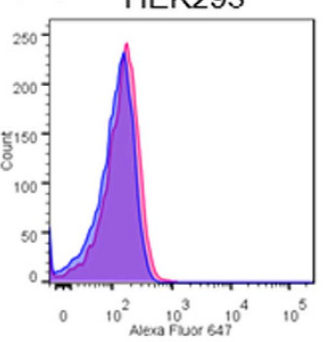

TE-11

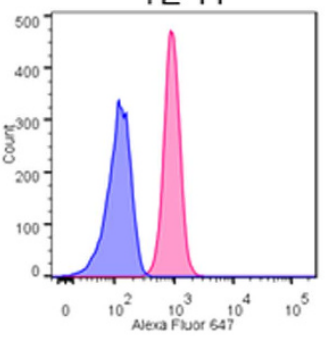

(C)

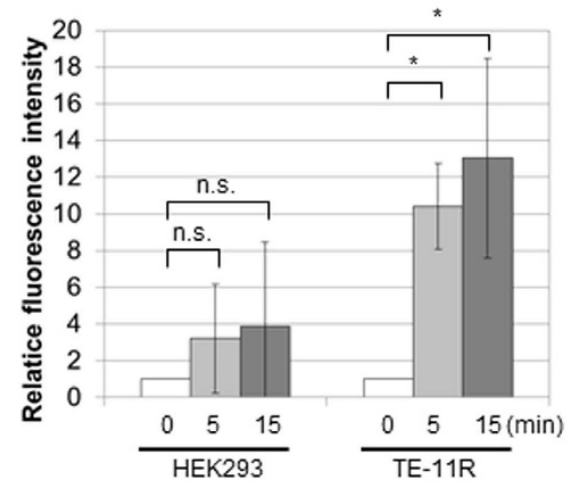

Exposure time

(peptide concentration: $10 \mu \mathrm{M}$ )
TE-5

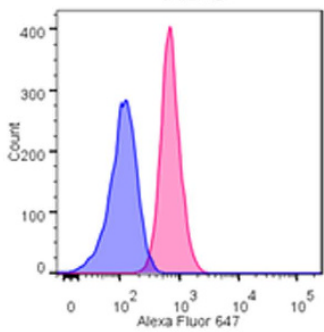

TE-5R

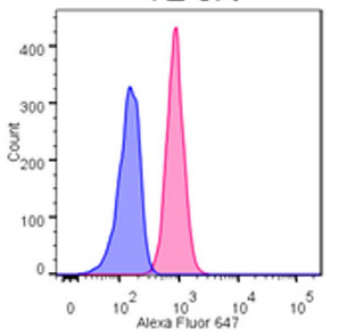

(D)

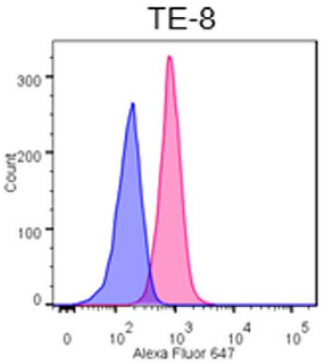

TE-11R
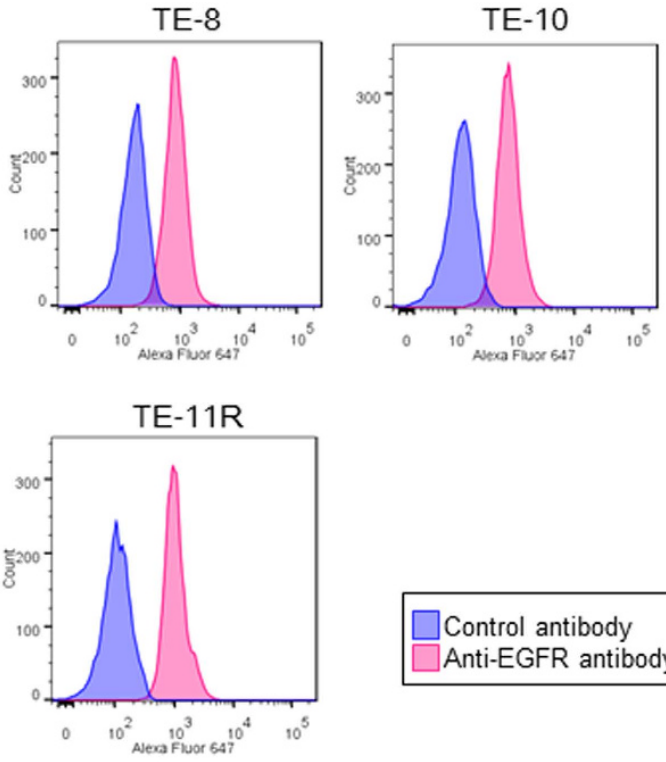

$\square$ Control antibody

Anti-EGFR antibody

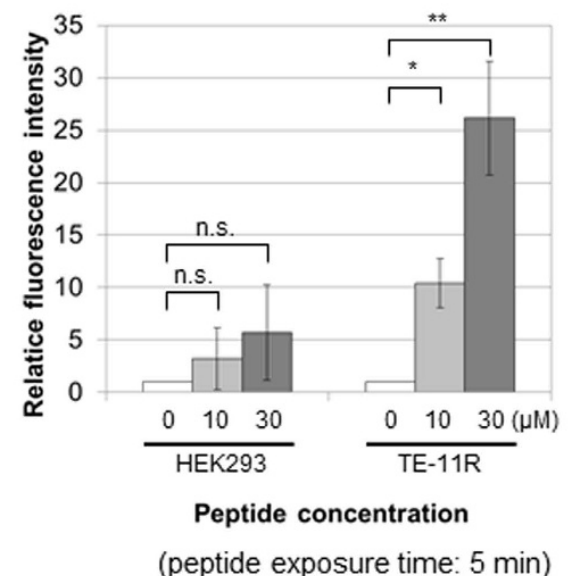

Figure 2. EGFR expression of OSCC cells and affinity of EGFR-binding peptide for OSCC cells. (A) EGFR expression levels in whole -cell lysates of HEK293 and OSCC cells (TE-5, TE-8, TE-10, TE-11, TE-5R, and TE-11R). The EGFR protein expression level was determined by Western blotting. $\beta$-Actin served as a loading control. EGFR protein was highly expressed in all OSCC cells, while its level was very low in HEK293 cells. (B) EGFR expressions on the cell surface of HEK293 and OSCC cells. Cells were stained with AlexaFluor 647-conjugated monoclonal anti-EGFR antibody or IgG isotype control antibody. The Fluorescence intensity determined by flow cytometry showed that EGFR was expressed on the surface of OSCC cells, whereas HEK293 cells minimally expressed it. (C,D) Affinity of EGFR-binding peptide for HEK293 and TE-11R cells. They were exposed to Fluorescein (FLC)-labelled EGFR-binding peptide, and the fluorescence intensity was assessed by flow cytometry. The Affinity of EGFR-binding peptide for cell membranes of TE-11R cells is increasing in time(C) and dose- (D) dependent manners, but not in HEK293 cells. The assays were repeated three times, and data are shown as the mean \pm SD. n.s.: not significant, ${ }^{\star} \mathrm{P}<0.05$, ${ }^{* *} \mathrm{P}<0.01$ vs. untreated ( 0 minutes) cells. 
HEK293

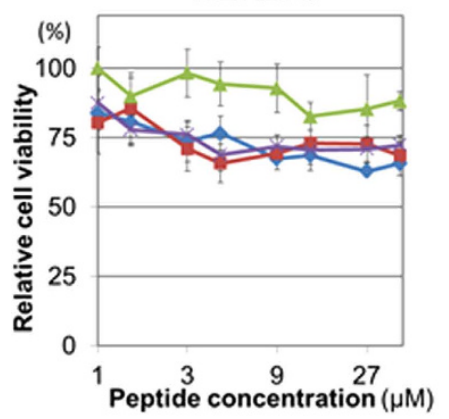

TE-10

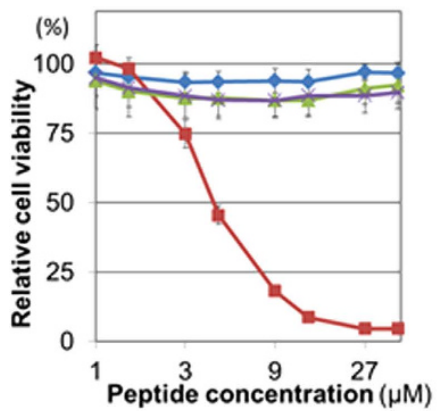

TE-5

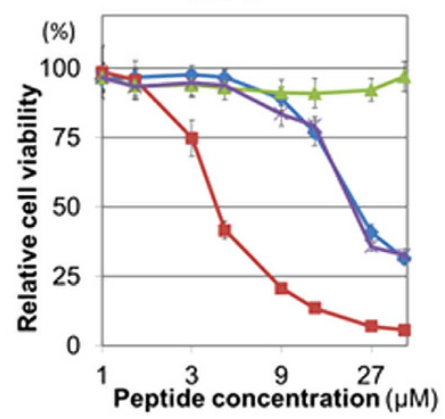

TE-8

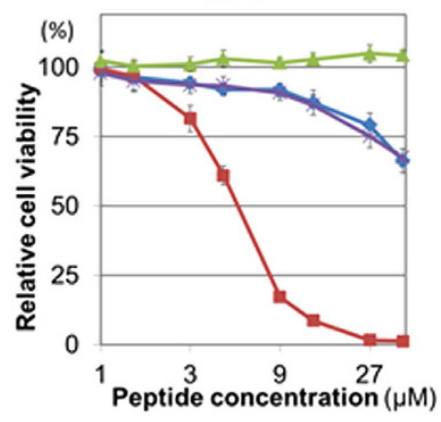

TE-11

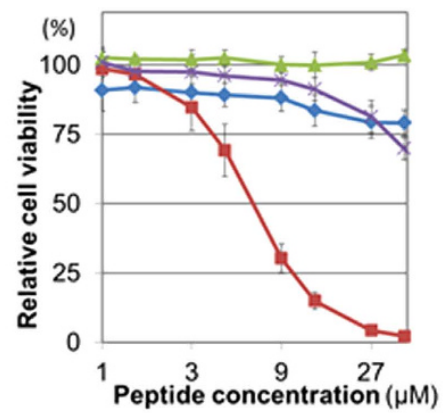

TE-5R

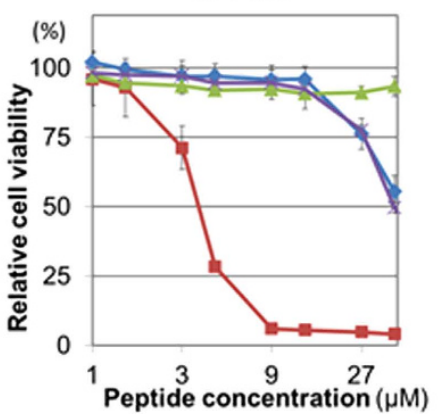

TE-11R

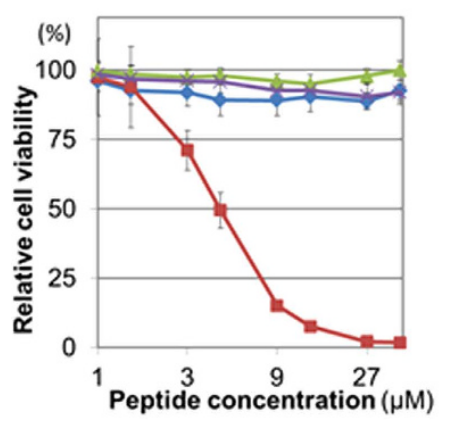

-EGFR-binding peptide

$\longrightarrow$ Lytic peptide

$\longrightarrow$ Co-administration of EGFR-binding peptide and Lytic peptide

- EGFR(2R)-lytic hybrid peptide

Figure 3. Cytotoxicity of EGFR(2R)-lytic hybrid peptide against OSCC cells in vitro. OSCC and control HEK293 cells were cultured with various concentrations $(0-50 \mu \mathrm{M})$ of the EGFR-binding peptide fragment alone, lytic peptide fragment alone, co-administration of EGFR-binding peptide and lytic peptide fragments, or EGFR(2R)-lytic hybrid peptide for 30 minutes, and cell viability was measured with the WST-1 assay. A viability of $100 \%$ was defined as the amount of absorption at $450 \mathrm{~nm}$ found in control cells. Each point represents the mean \pm S.D. (bars) from experiments conducted in sextuplicate, and the assays were repeated three times. Note that EGFR(2R)-lytic hybrid peptide exhibits a potent cytotoxicity against all OSCC cells at very low concentrations of less than $10 \mu \mathrm{M}$.

Tolerability of EGFR(2R)-lytic hybrid peptide. We assessed the toxicity of EGFR(2R)-lytic hybrid peptide in mice. Its administration led to no apparent gross abnormalities of the skin or abdominal cavities of mice. In addition, no histological damage was evident in organs (e.g., lung, kidney, oesophagus, and liver) (Fig. 6), which have been reported to express high levels of EGFR. Furthermore, neither significant haematological adverse effects nor weight loss was detected (Table 1, Supplementary Fig. S2).

Additionally, we tried to expose EGFR(2R)-lytic hybrid peptide on the stratified squamous epithelium. To this end, we conducted an organotypic 3D culture using human skin or oesophageal cells. Even though EGFR(2R)-lytic hybrid peptide was exposed to the stratified squamous epithelium, it did not cause any apparent histological damage (Fig. 7). 
(A)

\section{0 min $\quad 5$ min}

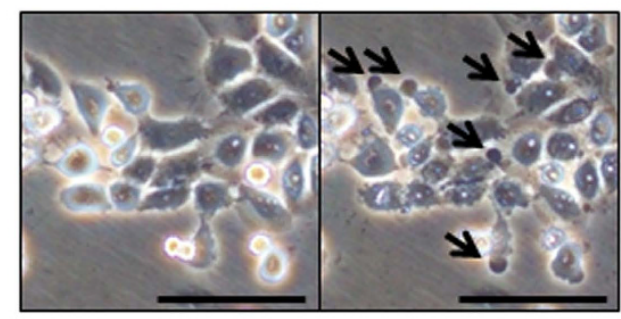

(B)

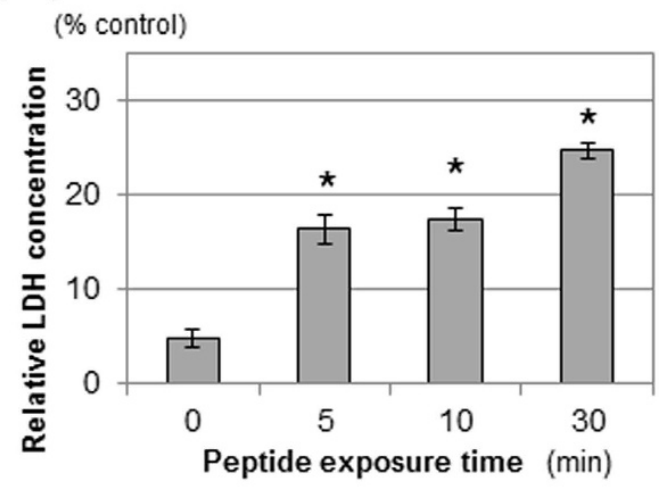

(C)

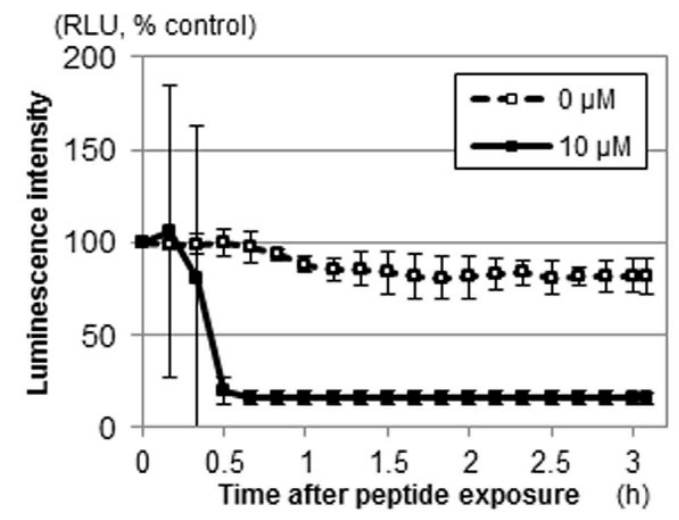

Figure 4. Rapid disintegration of TE-11R cell membranes after exposure to EGFR(2R)-lytic hybrid peptide in vitro. (A) Phase-contrast images of TE-11R cells after exposure to EGFR(2R)-lytic hybrid peptide $(10 \mu \mathrm{M})$. Extravasation from cell membranes (arrows) occurred at 5 minutes after exposure to EGFR(2R)-lytic hybrid peptide. Scale bar, $100 \mu \mathrm{m}$. (B) Extracellular LDH concentrations in culture medium after exposure to EGFR(2R)-lytic hybrid peptide. The relative LDH concentration (\% of positive control with Triton X-100) was calculated. A time-dependent increase of extracellular LDH levels was found after exposure to EGFR(2R)lytic hybrid peptide $(10 \mu \mathrm{M})$. Each point represents the mean \pm S.D. (bars) from experiments conducted in sextuplicate. ${ }^{\star} \mathrm{P}<0.001$ vs. untreated $(0$ minutes) cells. $(\mathbf{C})$ The real-time cytotoxicity determined by ATP levels in TE-11R cells treated with EGFR(2R)-lytic hybrid peptide. TE-11R cells were transiently transfected with pGL4.50 vector and exposed with (right panel) or without (left panel) EGFR(2R)-lytic hybrid peptide $(10 \mu \mathrm{M})$ for the indicated durations. Each point represents the mean \pm S.D. (bars) from three cells. Note that rapid elimination of ATP was observed in the cells treated with EGFR(2R)-lytic hybrid peptide, but not in those treated without the peptide. These results indicate the rapid disintegration of the cell membrane and consequent cell death after exposure to EGFR(2R)-lytic hybrid peptide.

\section{Discussion}

In the present study, we showed that all the OSCC cells we used expressed high levels of EGFR on their surface. The EGFR-binding fragment of EGFR(2R)-lytic hybrid peptide showed affinity for OSCC cells, but not HEK293 
(A)

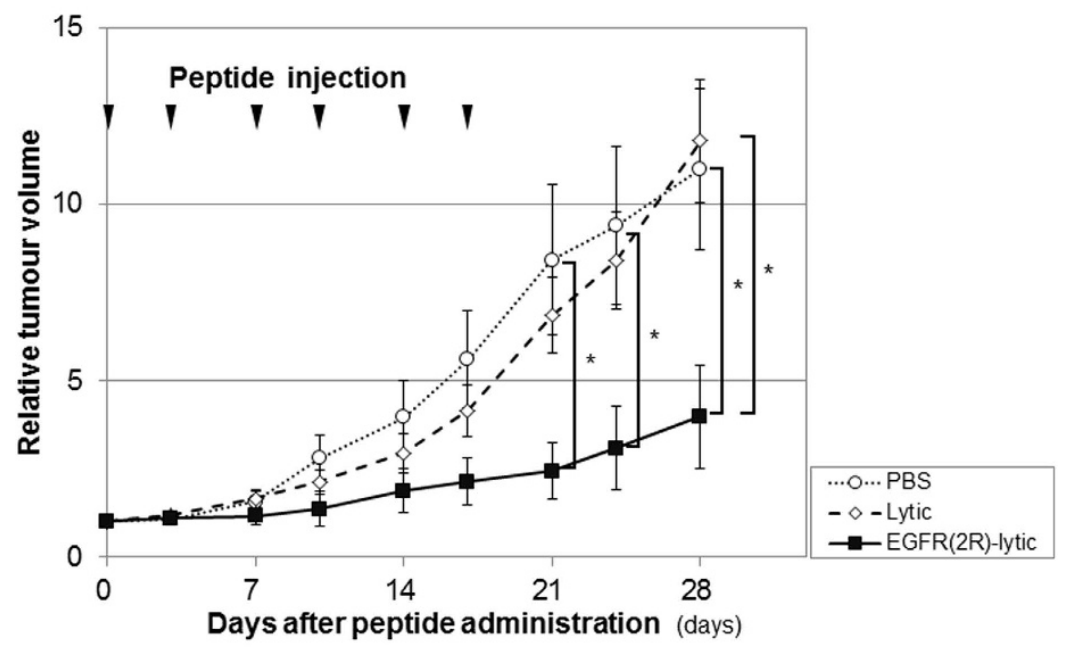

(B)
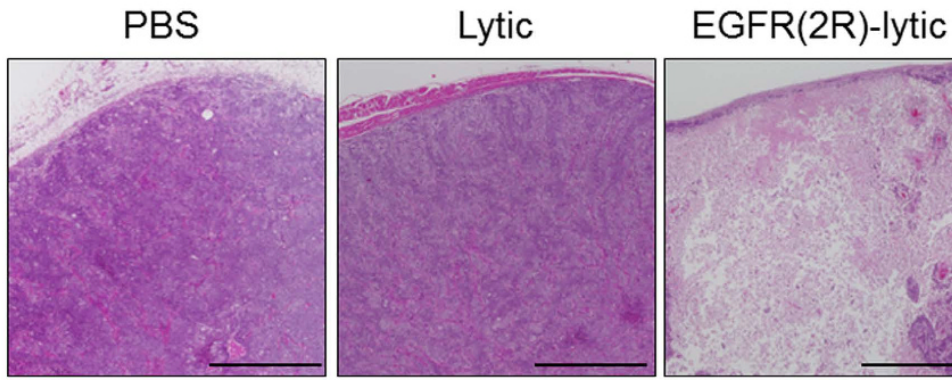

Figure 5. An anti-tumour effect of $\operatorname{EGFR(2R)-lytic~hybrid~peptide~against~xenografted~OSCC~tumours.~}$ (A) Time-course volumes of xenografted tumours in vivo. TE-11R cells were allowed to grow for 5 weeks, and either PBS (O), lytic peptide $(\diamond: 4 \mathrm{mg} / \mathrm{kg})$, or EGFR(2R)-lytic hybrid peptide ( $\mathbf{\square}: 4 \mathrm{mg} / \mathrm{kg}$ ) was intravenously injected twice a week for a total of six doses, as indicated by the arrowheads $(n=5)$. The relative tumour volumes on day 28 in proportion to the volume on day 0 of the PBS, lytic peptide, and EGFR(2R)-lytic hybrid peptide groups were 11.0, 11.8 and 4.0, respectively. Each point represents the mean \pm SEM (bars). ${ }^{\star} \mathrm{P}<0.05$ vs. PBS-treated or lytic peptide-treated mice. (B) H\&E staining of representative tumours in each group. Note that EGFR(2R)-lytic hybrid peptide induced potent tissue injury but not PBS or lytic peptide. Scale bar, $1 \mathrm{~mm}$.

cells. EGFR(2R)-lytic hybrid peptide showed a potent cytotoxicity as well as anti-tumour effect against OSCC cells both in vitro and in vivo. Furthermore, the administration of EGFR(2R)-lytic hybrid peptide did not cause any adverse haematological or histological effects in mice. We mainly used TE-11R cells to assess the cytotoxic or anti-tumour effect of EGFR(2R)-lytic hybrid peptide, because TE-11R cells are highly transformed cells with tumourigenicity ${ }^{18}$.

In this study, normal oesophageal keratinocytes were not used in our in vitro experiments. Normal oesophageal keratinocytes are usually cultured in medium with a low calcium concentration (e.g., keratinocyte serum-free medium), in which these keratinocytes grow as undifferentiated basal cells ${ }^{19}$. Indeed, cultured normal oesophageal keratinocytes express high levels of cytokeratin 14 (protein expressed in the basal layers of the oesophageal stratified epithelium) but low levels of cytokeratin 13 and involucrin (proteins expressed in the suprabasal layers of the oesophageal stratified epithelium $)^{19}$. Thus, cultured normal oesophageal keratinocytes are cells that enrich basal cells, in which EGFR is expressed ${ }^{20}$. Accordingly, EGFR expression in cultured oesophageal keratinocytes is high, and we could not show the difference between OSCC cells and normal oesophageal keratinocytes regarding the cytotoxicity with EGFR(2R)-lytic hybrid peptide in our in vitro experiments. Alternatively, the safety of EGFR(2R)-lytic hybrid peptide for the normal oesophagus was investigated by in vivo and organotypic $3 \mathrm{D}$-culture experiments.

We showed that EGFR(2R)-lytic hybrid peptide had a higher cytotoxicity than the lytic peptide fragment. According to the report of Papo et al. the lytic peptide fragment forms a random coil structure in a solution, in which its ability to cause cell membrane disruption is weak ${ }^{21}$. However, the form of lytic peptide can be changed to an $\alpha$-helical structure when it is attracted to the cell surface by static electricity due to the lipid bilayer ${ }^{22,23}$ and it exerts enhanced cytotoxicity with cell membrane disruption ${ }^{21}$. Notably, the EGFR expression level on the cell surface affects the cytotoxicity of EGFR-lytic hybrid peptide ${ }^{15}$, suggesting that the EGFR-binding peptide 


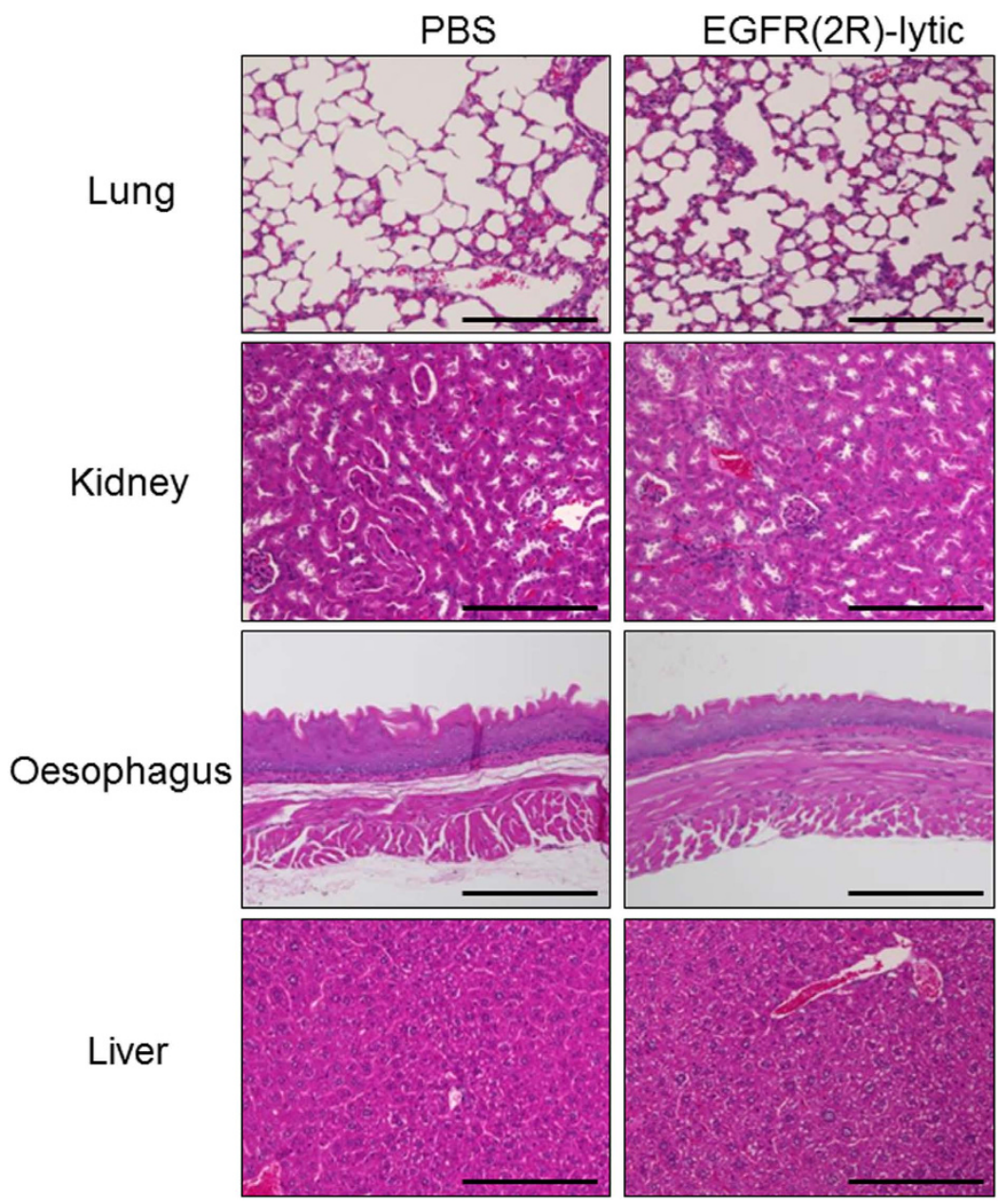

Figure 6. Tolerability of EGFR(2R)-lytic hybrid peptide in mice. H\&E staining of the lung, kidney, oesophagus, and liver of BALB/c mice after 6-times intravenous administration of EGFR(2R)-lytic hybrid peptide $(4 \mathrm{mg} / \mathrm{kg})$. Peptides were intravenously injected twice a week for a total of six doses. Note that the administration of EGFR(2R)-lytic hybrid peptide did not affect those organs. Scale bar, $200 \mu \mathrm{m}$.

\begin{tabular}{|l|c|c|c|c|}
\hline & EGFR(2R)-lytic & Lytic & PBS & P \\
\hline WBC $\left(\times 10^{3} / \mu \mathrm{L}\right)$ & $2.5 \pm 0.7$ & $2.5 \pm 0.2$ & $3.1 \pm 1.0$ & 0.341 \\
\hline $\mathrm{RBC}\left(\times 10^{4} / \mu \mathrm{L}\right)$ & $1002.2 \pm 62.1$ & $909.4 \pm 138.4$ & $907.8 \pm 216.3$ & 0.550 \\
\hline $\mathrm{Hb}(\mathrm{g} / \mathrm{dL})$ & $16.1 \pm 0.1$ & $15.8 \pm 0.7$ & $15.3 \pm 2.1$ & 0.593 \\
\hline $\mathrm{Hct}(\%)$ & $52.9 \pm 3.3$ & $49.1 \pm 7.4$ & $47.6 \pm 10.5$ & 0.549 \\
\hline $\mathrm{Plt}\left(\times 10^{4} / \mu \mathrm{L}\right)$ & $81.5 \pm 4.6$ & $77.0 \pm 11.6$ & $69.8 \pm 26.0$ & 0.546 \\
\hline
\end{tabular}

Table 1. Haematological assessment after the administration of EGFR(2R)-lytic hybrid peptide to mice. EGFR(2R)-lytic hybrid peptide ( $4 \mathrm{mg} / \mathrm{kg}$ ) was intravenously injected into BALB/c mice twice a week for a total of six doses. Blood samples were obtained one day after the final intravenous injection of EGFR(2R)lytic hybrid peptide. P-values were assessed by analysis of variance (ANOVA). Data are presented as the mean \pm S.D. $(n=5)$. Note that $\operatorname{EGFR}(2 R)$-lytic hybrid peptide administration did not cause any haematological abnormalities in mice.

fragment acts as an anchor to EGFR-expressing cells, and binding of the EGFR-binding fragment with EGFR on the cell surface contributes to change the lytic peptide fragment structurally and increase membranolytic cytotoxicity. Indeed, EGFR(2R)-lytic hybrid peptide showed high-level cytotoxicity against OSCC cells, whereas it was subtle when EGFR-binding peptide and lytic peptide fragments were not hybridized (co-administration of EGFR-binding peptide and lytic peptide fragments). These results indicate that the hybridisation of EGFR-binding peptide and lytic peptide fragments plays a key role to enhance the membranolytic cytotoxicity of lytic peptide fragments.

The therapeutic effect of existing EGFR-targeting therapy on ESCC is not sufficient. In OSCC, EGFR is frequently expressed $^{9}$, while the mutation rate is very low $(1.1 \%)^{24}$. On the other hand, gene mutations and amplifications of EGFR downstream signalling pathways are frequently noted $(78.6 \%)^{24}$. The therapeutic effect of existing 


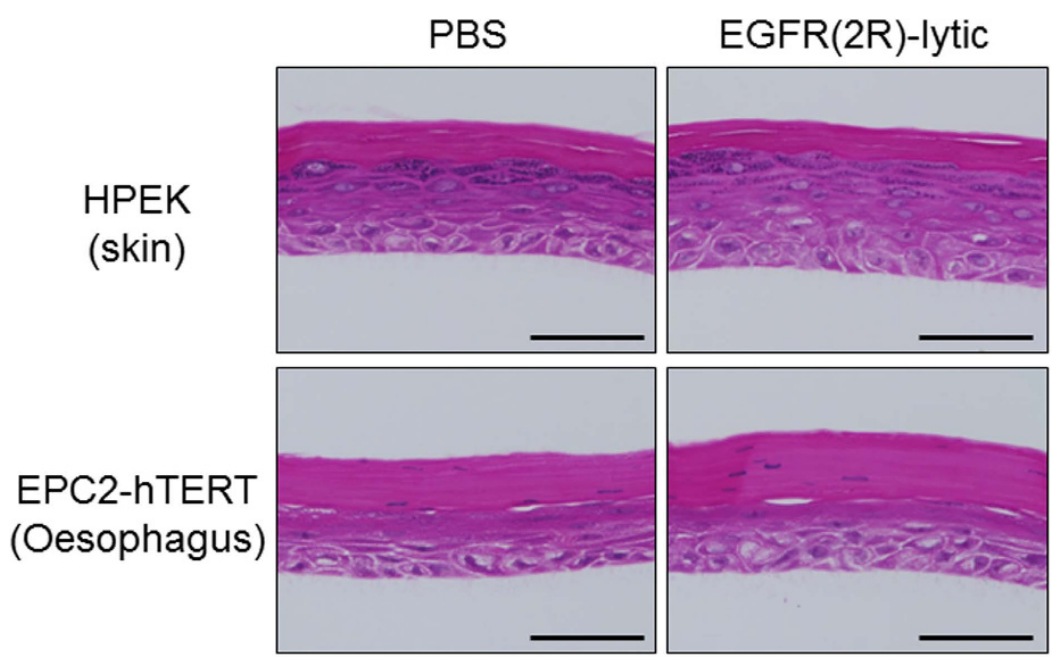

Figure 7. Toxic effect of EGFR(2R)-lytic hybrid peptide against stratified squamous epithelium in 3D culture. EGFR(2R)-lytic hybrid peptide $(1 \mathrm{mg} / \mathrm{mL})$ or PBS was added to the apical site of $3 D$-culture cell sheets consisting of HPEK (human skin primary keratinocytes) or EPC2-hTERT (immortalized human oesophageal keratinocytes) for 30 minutes. Note that exposure to EGFR(2R)-lytic hybrid peptide did not adversely affect stratified squamous epithelium derived from human skin or oesophageal keratinocytes. H\&E staining. Scale bar, $50 \mu \mathrm{m}$.

EGFR-targeted therapies is achieved by blocking EGFR signalling in the tumour. Therefore, it is influenced by gene alteration of EGFR as well as EGFR downstream signal cascades. For example, in non-small lung cancer, response rates of EGFR-TKI are more favourable in patients with than without EGFR mutations ${ }^{25}$. Moreover, in colon cancer, the therapeutic effects of anti-EGFR antibody are weaker in patients with mutations of molecules downstream of EGFR than those in patients without such mutations ${ }^{26,27}$. These results suggest that the low response rate to existing EGFR-targeted therapies in OSCC patients might be due to the low frequency of EGFR mutation as well as high frequency of gene alteration of EGFR downstream signalling pathways. In this study, the anti-tumour effect of EGFR(2R)-lytic hybrid peptide is considered to depend on cell membranous EGFR expression, but not on the intracellular EGFR signalling cascades, because the pretreatment of OSCC cells with Erlotinib did not affect the cytotoxicity of EGFR(2R)-lytic hybrid peptide (Supplementary Fig. S3). Taken together, we believe that EGFR-targeted therapy using EGFR(2R)-lytic hybrid peptide is a valid strategy against OSCC.

In this study, EGFR(2R)-lytic hybrid peptide induced rapid disintegration of the cell membrane and ATP depletion in OSCC cells. Cell membrane damage with LDH leakage indicates necrotic cell death ${ }^{28}$, whereas ATP depletion indicates the loss of functional integrity of living cells ${ }^{29}$. Although our data could not determine whether cell membrane disintegration precedes or follows ATP depletion, EGFR(2R)-lytic hybrid peptide could eliminate OSCC cells effectively in vitro.

EGFR(2R)-lytic hybrid peptide also showed a cytotoxic effect against 5-FU-resistant TE-5R and TE-11R cells, which were established by step-wise treatment with continuously increasing concentrations of 5 -FU ${ }^{18,30}$. The mechanism of 5-FU resistance in TE-5R cells is based on a gene amplification of dihydropyrimidine dehydrogenase, a 5-FU-degrading enzyme ${ }^{30}$. On the other hand, the mechanisms of 5-FU resistance in TE-11R cells have not been fully elucidated; however, TE-11R cells show marked tumorigenicity ${ }^{18}$. In this study, we showed that EGFR expression on TE-5R and TE-11R cells was comparable with that on parental TE-5 and TE-11 cells. To our knowledge, there have been no reports regarding the relationship between 5-FU resistance and EGFR expression in OSCC. We suggest that EGFR(2R)-lytic hybrid peptide could be effective against OSCC cells irrespective of 5 -FU resistance as long as EGFR is overexpressed on their surface.

We suspect that EGFR(2R)-lytic hybrid peptide affects noncancerous cells that express EGFR (e.g., liver hepatocytes, respiratory epithelial cells of bronchus and lung, cells in kidney tubules, and epidermal keratinocytes of oesophagus and $\operatorname{skin}^{31,32}$ ); however, it did not show marked toxicity against those normal tissues in mice. Although the EGFR-binding peptide sequence was originally determined by phage display screening using human recombinant $\mathrm{EGFR}^{17}$, it was further modified to improve the biomolecular interaction with human EGFR $^{16}$. The homology of the extracellular domain of human and mouse EGFR is $89 \%$ (Supplementary Fig. S4), and the EGFR-binding peptide fragment could certainly bind to mouse cells (Supplementary Fig. S5).

A possible reason why EGFR(2R)-lytic hybrid peptide has more preferential cytotoxicity against cancer cells than noncancerous cells is suggested as follows: The lytic peptide fragment has a net positive charge $\mathrm{e}^{21}$. On the other hand, cancer cells carry more negative charges due to the presence of negatively charged phosphatidylserine than noncancerous cells ${ }^{33}$. Accordingly, the lytic peptide fragment itself has more affinity toward cancerous than noncancerous cells ${ }^{21}$. Moreover, the EGFR-binding peptide fragment is considered to contribute to bind the lytic peptide fragment with high EGFR-expressing OSCC cells and help with the $\alpha$-helical structural change of the lytic peptide fragment, as we described. Thus, the selectivity of EGFR(2R)-lytic hybrid peptide is suggested to be enhanced in OSCC cells. 
As a limitation, the possibility that EGFR(2R)-lytic hybrid peptide might interact with other cell surface proteins cannot be excluded. To conduct a first-in-human clinical trial, further examination regarding absorption, distribution, metabolism, and excretion as well as the metabolic stability of this peptide should be performed.

In conclusion, we conducted a preclinical study of using EGFR(2R)-lytic hybrid peptide against OSCC, and showed the validity of this peptide as a novel EGFR-targeted therapy.

\section{Methods}

Cell lines and cell culture. Human OSCC cell lines (TE-5, TE-8, TE-10, and TE-11) were obtained from Riken BioResource Center (Ibaragi, Japan) ${ }^{34}$. TE-5R and TE-11R cells, which are derived from TE-5 and TE-11 cells, respectively, are 5-FU-resistant OSCC cells that were established via exposure to parental cells with gradually increasing concentrations of 5-FU ${ }^{18,30}$. All OSCC cells were cultured in RPMI1640 medium (Life Technologies Corp., Grand Island, NY, USA), supplemented with 10\% foetal bovine serum (FBS; Life Technologies.), $100 \mu \mathrm{g} /$ $\mathrm{mL}$ of streptomycin, and 100 units/mL of penicillin (Life Tech.) at $37^{\circ} \mathrm{C}$ in a $5 \% \mathrm{CO}_{2}$ incubator.

HEK293, a cell line derived from human embryonic kidney cells ${ }^{35}$, served as low EGFR-expressing cells ${ }^{36,37}$. HEK293 was purchased from DS Pharma Biomedical Co., Ltd. (Osaka, Japan) and cultured in DMEM medium (Life Tech.), supplemented with $10 \% \mathrm{FBS}, 100 \mu \mathrm{g} / \mathrm{mL}$ of streptomycin, and $100 \mathrm{units} / \mathrm{mL}$ of penicillin at $37^{\circ} \mathrm{C}$ in a $5 \% \mathrm{CO}_{2}$ incubator.

Peptides. The following peptides were purchased from American Peptide Company Inc., (Sunnyvale, CA, USA) or Sigma-Aldrich Co., (St. Louis, MO, USA). Bold and italic letters are D-amino acids.

1. EGFR(2R)-lytic hybrid peptide: YRWYGYTPQNVIGGGKLLLKLLKKLLKLLKKK (American Peptide)

2. EGFR-binding peptide: YRWYGYTPQNVI (Sigma-Aldrich)

3. Lytic peptide (without EGFR-binding sequence): KLLLKLLKKLLKLLKKK (American Peptide)

4. Fluorescein (FLC)-labelled EGFR-binding peptide: FLC-YRWYGYTPQNVI (labelled with FLC at its N-terminal, Sigma-Aldrich)

Measurement of EGFR expression. EGFR expression of the cells used in this study was measured by Western blotting and flow cytometry.

Whole-cell lysates were collected and Western blotting was performed as described previously ${ }^{18}$. Primary antibodies and the titres used in this study were as follows: rabbit monoclonal anti-EGFR antibody (D38B1, \#4267, Cell Signaling Technology, Inc., Danvers, MA, USA; 1:1,000), rabbit monoclonal anti- $\beta$-Actin antibody (13E5, \#5125, Cell Signaling; 1:5,000). $\beta$-Actin served as loading controls for whole-cell lysates.

EGFR expression on the cell surface was detected with flow cytometry by measuring the fluorointensity of dye-conjugated anti-EGFR antibody which binds to cell-surface EGFR with a minor modification from a previous report $^{38}$. In brief, cells were washed twice with PBS, and they were stained for 30 minutes on ice with AlexaFluor 647-conjugated anti-EGFR or control antibodies in 2\% FBS in PBS. Then, cells were washed twice with PBS and analysed with a flow cytometer (BD LSRFortessa Analyzer; BD Biosciences, San Jose, CA, USA). Antibodies and the titres were as follows: AlexaFluor 647-conjugated monoclonal anti-EGFR antibody (D38B1, \#5588, Cell Signaling; 1:50), rabbit IgG isotype control (Alexa Fluor 647 Conjugate) antibody (\#3452, Cell Signaling; 1:50). Collected data were analysed using FlowJo X software (Tree Star, Inc., Ashland, OR, USA).

Affinity of EGFR-binding peptide to cell surface EGFR. To determine the affinity of the EGFR-binding peptide fragment to the surface of OSCC cells, the FLC-labelled EGFR-binding peptide fragment was incubated with TE-11R cells and control HEK293 cells, and the fluorointensity was measured with a flow cytometer. In brief, these cells $\left(1 \times 10^{6}\right.$ cells $\left./ \mathrm{mL}\right)$ were stained with the FLC-labelled EGFR-binding peptide fragment at the indicated times and/or concentrations on ice. After staining, these cells were washed twice with PBS, resuspended in $400 \mu \mathrm{L}$ of ice-cold PBS, and the fluorointensity of FLC was detected through a 530/30 band-pass filter after excitation with a 488-nm blue laser.

WST-1 assays. The cytotoxic effect of the EGFR-binding peptide fragment alone, lytic peptide fragment alone, co-administration of EGFR-binding peptide fragment with lytic peptide fragment, or EGFR(2R)-lytic hybrid peptide on OSCC cells was determined by the WST-1 assay (Roche Applied Science, Upper Bavaria, Germany) following the manufacturer's instructions. Cells $\left(2 \times 10^{4}\right.$ cells $)$ were seeded in 96 -well plates, and exposed to the indicated concentrations of peptides for 30 minutes. All data were obtained in sextuplicate with a multi-well plate reader as $\mathrm{A}_{450}-\mathrm{A}_{630}$ (Infinite 200 pro, Tecan, Männedorf, Switzerland). Data are presented as the mean \pm standard deviation (SD).

LDH cytotoxicity assay. LDH is a cytosolic enzyme and is released from cells when plasma membranes are damaged $^{39}$. Therefore, we measured the extracellular LDH concentration with the LDH Cytotoxicity Detection Kit (Takara Bio, Otsu, Japan) to detect cell membrane damage induced by EGFR(2R)-lytic hybrid peptide. In brief, OSCC cells $\left(2 \times 10^{4}\right.$ cells $)$ were plated in 96-well plates and, after 24-hour incubation, the media was changed to RPMI1640 (Life Tech.) without FBS, and they were exposed to the indicated concentrations of EGFR(2R)-lytic hybrid peptide in triplicate for 30 minutes. The media from each sample was transferred into a 96-well plate and incubated with a reaction mixture including tetrazolium salt, and the $\mathrm{LDH}$ concentration was measured as $\mathrm{A}_{492}$ $-\mathrm{A}_{630}$ on a multi-well plate reader.

ATP real-time bioluminescence assay. ATP is the most important chemical energy reservoir in cells, and cell death results in a rapid ATP decrease ${ }^{29}$. ATP levels can be determined by assessing ATP bioluminescence 
with the luciferin-luciferase reaction ${ }^{40}$. Therefore, this assay is used to assess real-time cell viability ${ }^{41}$. In this study, TE-11R cells were transiently transfected with firefly luciferase-containing reporter plasmids of the cytomegalovirus promoter (pGL4.50, Promega Corp., Madison, WI, USA) using Lipofectamine LTX (Life Tech.) according to the manufacturer's protocol, and bioluminescence images were obtained with the LUMINOVIEW LV200 luminescence imaging system ( $40 \times$ objective; 5 -minute intervals; 10 -second exposure; Olympus Corp., Tokyo, Japan) after incubation with or without EGFR(2R)-lytic hybrid peptide. The dish was kept at $37^{\circ} \mathrm{C}$ in a humidified chamber and images were taken while observing promoter activity after the addition of D-luciferin $(500 \mu \mathrm{M}$; Promega). The regions of interest (ROIs) were selected on each cell and the luminescence intensity was measured every five minutes using LV200. Data were analysed by AQUACOSMOS ver. 2.6 software (Hamamatsu Photonics, Shizuoka, Japan).

In vivo experiments. All experiments conformed to the relevant regulatory standards and were approved by the Institutional Animal Care and Use Committee of Kyoto University (Med Kyo 14523).

Xenograft transplantation was conducted as described previously ${ }^{18}$. Briefly, TE-11R cells $\left(10 \times 10^{6}\right)$ were suspended in $50 \%$ matrigel (BD Biosciences), followed by their subcutaneous implantation into the dorsal skin of nude male mice ( 9 weeks of age; CLEA Japan, Inc., Tokyo, Japan). Xenografted tumours were used for the following experiments and divided into 3 groups when they had reached a tumour volume of about $50-180 \mathrm{~mm}^{3}$ at 40 days after injection. Tumours were free of evident necrosis at the beginning of injection. EGFR(2R)-lytic hybrid peptide $(4 \mathrm{mg} / \mathrm{kg}$ ), lytic peptide $(4 \mathrm{mg} / \mathrm{kg})$, or PBS was administered intravenously via the tail vein of mice twice a week for a total of six doses. The first administration day was defined as day 0 . The tumours were monitored twice a week with a calliper, and the tumour volume $\left(\mathrm{mm}^{3}\right)$ was calculated using the following formula: length $\mathrm{x}$ width $^{2} \times 0.5$. The body weights were also monitored twice a week. The Relative tumour volume is shown as the Tumour volume on the indicated day/Tumour volume on day 0 , and is presented as the mean \pm standard error of the mean (SEM).

To assess the tolerability of EGFR(2R)-lytic hybrid peptide in vivo, we conducted a haematological and histological examination. We searched for anomalies in the abdominal cavity and assessed the appearance of the skin of mice, and then obtained blood and tissue samples (lung, heart, spleen, kidney, oesophagus, and liver) after 6-times administration of EGFR(2R)-lytic hybrid peptide, lytic peptide, or PBS to BALB/c mice. The body weights were monitored twice a week. Blood samples were obtained under sedation 24 hours after the final intravenous injection of EGFR(2R)-lytic hybrid peptide. Then, mice were painlessly sacrificed under appropriate anaesthesia with the inhalation of isoflurane (Escain, Mylan Pharmaceuticals, Tokyo, Japan) and cervical dislocation, and tissue samples were collected in $10 \%$ formaldehyde phosphate buffer solution (Wako Pure Chemical Industries, Ltd.) for histopathology $(n=5)$.

Tolerability assessments with three-dimensional culture. To assess the safety of handling EGFR(2R)-lytic hybrid peptide in clinical situations such as preparing injection solution, we conducted an additional assessment using a three-dimensional (3D) culture model of human cells. 3D culture was established with the 3D Keratinocyte Starter Kit (3D-HPEK-50, CELLnTEC Advanced Cell Systems AG, Bern, Switzerland) following the manufacturer's instructions. In brief, $2 \times 10^{5}$ human primary skin keratinocytes ${ }^{42}$ (HPEK, CELLnTEC) and human oesophageal keratinocytes immortalized with human telomerase reverse transcriptase $(\text { EPC2-hTERT })^{43}$ were seeded in the insert for 3 days with CnT-PCT medium $(400 \mu \mathrm{L})$, and then the medium was changed to 3D Prime differentiation medium inside as well as outside the insert. After overnight culture with the 3D Prime differentiation medium, 3D cultures were initiated by aspirating all the medium from inside the insert and replacing only the outside medium with fresh 3D Prime differentiation medium up to the level of the membrane, to dry the surface within the inserts. After culture with the appropriate medium for 14 days, EGFR(2R)-lytic hybrid peptide ( $1 \mathrm{mg} / \mathrm{mL}$, same concentration as used in mouse experiments) or PBS was added to the apical site of 3D-culture cell sheets, and cell sheets were harvested after exposure to EGFR(2R)-lytic hybrid peptide for 30 minutes. $3 \mathrm{D}$ cultures were fixed with $4 \%$ paraformaldehyde phosphate buffer solution (Wako Pure Chemical Industries, Ltd.) for histopathology $(n=3)$.

Statistical analyses. Data are presented as the mean \pm standard deviation of the sextuplicate experiments, unless otherwise noted. Data were analysed using the 2-tailed Student's $t$-test between two groups, or analysis of variance (ANOVA) between three groups. $P<0.05$ was considered significant. All statistical analyses were performed with SPSS 19 for Windows (SPSS Inc., Chicago, IL, USA).

\section{References}

1. Kamangar, F., Dores, G. M. \& Anderson, W. F. Patterns of cancer incidence, mortality, and prevalence across five continents: defining priorities to reduce cancer disparities in different geographic regions of the world. J Clin Oncol 24, 2137-2150 (2006).

2. Ancona, E. et al. Only pathologic complete response to neoadjuvant chemotherapy improves significantly the long term survival of patients with resectable esophageal squamous cell carcinoma: final report of a randomized, controlled trial of preoperative chemotherapy versus surgery alone. Cancer 91, 2165-2174 (2001).

3. Law, S., Fok, M., Chow, S., Chu, K. M. \& Wong, J. Preoperative chemotherapy versus surgical therapy alone for squamous cell carcinoma of the esophagus: a prospective randomized trial. J Thorac Cardiovasc Surg 114, 210-217 (1997).

4. National Comprehensive Cancer Network (NCCN) Clinical Practice Guidelines in Oncology: Esophageal and Esophagogastric Junction Cancers Version.3. (2015).

5. Kato, K. et al. A phase II study of nedaplatin and 5-fluorouracil in metastatic squamous cell carcinoma of the esophagus: The Japan Clinical Oncology Group (JCOG) Trial (JCOG 9905-DI). Esophagus 11, 183-188 (2014).

6. Iizuka, T. et al. Phase II evaluation of cisplatin and 5-fluorouracil in advanced squamous cell carcinoma of the esophagus: a Japanese Esophageal Oncology Group Trial. Jpn J Clin Oncol 22, 172-176 (1992).

7. Hayashi, K. et al. Phase II evaluation of protracted infusion of cisplatin and 5-fluorouracil in advanced squamous cell carcinoma of the esophagus: a Japan Esophageal Oncology Group (JEOG) Trial (JCOG9407). Jpn J Clin Oncol 31, 419-423 (2001). 
8. Muro, K. et al. A phase II study of single-agent docetaxel in patients with metastatic esophageal cancer. Ann Oncol 15, 955-959 (2004).

9. Laskin, J. J. \& Sandler, A. B. Epidermal growth factor receptor: a promising target in solid tumours. Cancer Treat Rev 30, 1-17 (2004).

10. Ozawa, S., Ueda, M., Ando, N., Shimizu, N. \& Abe, O. Prognostic significance of epidermal growth factor receptor in esophageal squamous cell carcinomas. Cancer 63, 2169-2173 (1989).

11. Crosby, T. et al. Chemoradiotherapy with or without cetuximab in patients with oesophageal cancer (SCOPE1): a multicentre, phase 2/3 randomised trial. Lancet Oncol 14, 627-637 (2013).

12. Lorenzen, S. et al. Cetuximab plus cisplatin-5-fluorouracil versus cisplatin-5-fluorouracil alone in first-line metastatic squamous cell carcinoma of the esophagus: a randomized phase II study of the Arbeitsgemeinschaft Internistische Onkologie. Ann Oncol 20, 1667-1673 (2009).

13. Ilson, D. H. et al. A phase 2 trial of erlotinib in patients with previously treated squamous cell and adenocarcinoma of the esophagus. Cancer 117, 1409-1414 (2011).

14. Dutton, S. J. et al. Gefitinib for oesophageal cancer progressing after chemotherapy (COG): a phase 3, multicentre, double-blind, placebo-controlled randomised trial. Lancet Oncol 15, 894-904 (2014).

15. Kohno, M. et al. A novel hybrid peptide targeting EGFR-expressing cancers. Eur J Cancer 47, 773-783 (2011).

16. Tada, N. et al. A single replacement of histidine to arginine in EGFR-lytic hybrid peptide demonstrates the improved anticancer activity. Biochem Biophys Res Commun 407, 383-388 (2011).

17. Li, Z. et al. Identification and characterization of a novel peptide ligand of epidermal growth factor receptor for targeted delivery of therapeutics. FASEB journal : official publication of the Federation of American Societies for Experimental Biology 19, 1978-1985 (2005).

18. Ohashi, S. et al. Preclinical validation of talaporfin sodium-mediated photodynamic therapy for esophageal squamous cell carcinoma. PloS one 9 , e103126 (2014).

19. Ohashi, S. et al. NOTCH1 and NOTCH3 coordinate esophageal squamous differentiation through a CSL-dependent transcriptional network. Gastroenterology 139, 2113-2123 (2010).

20. Miyawaki, M. et al. Enhanced phosphorylation of the epidermal growth factor receptor at the site of tyrosine 992 in esophageal carcinomas. APMIS 116, 1097-1106 (2008).

21. Papo, N. \& Shai, Y. New lytic peptides based on the D,L-amphipathic helix motif preferentially kill tumor cells compared to normal cells. Biochemistry 42, 9346-9354 (2003).

22. Sato, H. \& Feix, J. B. Peptide-membrane interactions and mechanisms of membrane destruction by amphipathic alpha-helical antimicrobial peptides. Biochim Biophys Acta 1758, 1245-1256 (2006).

23. Eiriksdottir, E., Konate, K., Langel, U., Divita, G. \& Deshayes, S. Secondary structure of cell-penetrating peptides controls membrane interaction and insertion. Biochim Biophys Acta 1798, 1119-1128 (2010).

24. Song, Y. et al. Identification of genomic alterations in oesophageal squamous cell cancer. Nature 509, 91-95 (2014).

25. Mok, T. S. et al. Gefitinib or carboplatin-paclitaxel in pulmonary adenocarcinoma. N Engl J Med 361, 947-957 (2009).

26. De Roock, W. et al. Effects of KRAS, BRAF, NRAS, and PIK3CA mutations on the efficacy of cetuximab plus chemotherapy in chemotherapy-refractory metastatic colorectal cancer: a retrospective consortium analysis. Lancet Oncol 11, 753-762 (2010).

27. Sorich, M. J. et al. Extended RAS mutations and anti-EGFR monoclonal antibody survival benefit in metastatic colorectal cancer: a meta-analysis of randomized, controlled trials. Ann Oncol 26, 13-21 (2015).

28. Korzeniewski, C. \& Callewaert, D. M. An enzyme-release assay for natural cytotoxicity. J Immunol Methods 64, 313-320 (1983).

29. Slater, K. Cytotoxicity tests for high-throughput drug discovery. Curr Opin Biotechnol 12, 70-74 (2001).

30. Kikuchi, O. et al. Novel 5-fluorouracil-resistant human esophageal squamous cell carcinoma cells with dihydropyrimidine dehydrogenase overexpression. Am J Cancer Res 5, 2431-2440 (2015).

31. Real, F. X. et al. Expression of epidermal growth factor receptor in human cultured cells and tissues: relationship to cell lineage and stage of differentiation. Cancer Res 46, 4726-4731 (1986).

32. Uhlen, M. et al. Proteomics. Tissue-based map of the human proteome. Science 347, 1260419 (2015)

33. Papo, N. \& Shai, Y. Host defense peptides as new weapons in cancer treatment. Cell Mol Life Sci 62, 784-790 (2005).

34. Nishihira, T., Hashimoto, Y., Katayama, M., Mori, S. \& Kuroki, T. Molecular and cellular features of esophageal cancer cells. J Cancer Res Clin Oncol 119, 441-449 (1993).

35. Harrison, T., Graham, F. \& Williams, J. Host-range mutants of adenovirus type 5 defective for growth in HeLa cells. Virology 77, 319-329 (1977).

36. Lill, N. L. et al. The evolutionarily conserved N-terminal region of $\mathrm{Cbl}$ is sufficient to enhance down-regulation of the epidermal growth factor receptor. J Biol Chem 275, 367-377 (2000).

37. Visser, G. D. \& Lill, N. L. The Cbl RING finger C-terminal flank controls epidermal growth factor receptor fate downstream of receptor ubiquitination. Exp Cell Res 311, 281-293 (2005).

38. Griffith, T. S. et al. Functional analysis of TRAIL receptors using monoclonal antibodies. J Immunol 162, 2597-2605 (1999).

39. Parhamifar, L., Andersen, H. \& Moghimi, S. M. In Nanotechnology for Nucleic Acid Delivery Vol. 948 Methods in Molecular Biology (eds Manfred Ogris \& David Oupicky) Ch. 2, 13-22 (Humana Press, 2013).

40. Moyer, J. D. \& Henderson, J. F. Ultrasensitive assay for ribonucleoside triphosphates in 50-1000 cells: Application to studies with pyrazofurin and mycophenolic acid. Biochem Pharmacol 32, 3831-3834 (1983).

41. Horibe, T. et al. Transfection efficiency of normal and cancer cell lines and monitoring of promoter activity by single-cell bioluminescence imaging. Luminescence 29, 96-100 (2014).

42. Lakatos, P. et al. 3-Aminobenzamide protects primary human keratinocytes from UV-induced cell death by a poly(ADP-ribosyl) ation independent mechanism. Biochimica et biophysica acta 1833, 743-751 (2013).

43. Harada, H. et al. Telomerase induces immortalization of human esophageal keratinocytes without p16INK4a inactivation. Mol Cancer Res 1, 729-738 (2003).

\section{Acknowledgements}

The authors are grateful for the technical assistance of Drs. Arong Gaowa and Ryohsuke Kurihara (Department of Pharmacoepidemiology, Kyoto University), Center for Anatomical, Pathological and Forensic Medical Researches and the Medical Research Support Center, Kyoto University Graduate School of Medicine. This study was supported by Grants-in-Aid for Young Scientist (A) (Grant No. 23680089), Scientific Research (B) (Grant No. 26290052), and Scientific Research (C) (Grant No. 25460926), and a Grant-in-Aid for JSPS Fellows (Grant No. 262741) from the Japan Society for the Promotion of Science (JSPS)-Japan. This research was supported by practical research for innovative cancer control from the Japan Agency for Medical Research and Development (AMED). This study was supported in part by a collaboration research fund from Olympus Corporation and Stella Pharma. 


\section{Author Contributions}

O.K. drafted the manuscript; S.O., T.H. and M.K. managed the study design and supervised data analysis. O.K., T.H., M.K. and Y.N. conducted data collection; S.O., T.H., M.K., S.M., T.C, M.M. and K.K. performed critical revision of the draft. All of the authors discussed the results and contributed to the manuscript.

\section{Additional Information}

Supplementary information accompanies this paper at http://www.nature.com/srep

Competing financial interests: The authors declare no competing financial interests.

How to cite this article: Kikuchi, O. et al. Novel EGFR-targeted strategy with hybrid peptide against oesophageal squamous cell carcinoma. Sci. Rep. 6, 22452; doi: 10.1038/srep22452 (2016).

(c) (i) This work is licensed under a Creative Commons Attribution 4.0 International License. The images or other third party material in this article are included in the article's Creative Commons license, unless indicated otherwise in the credit line; if the material is not included under the Creative Commons license, users will need to obtain permission from the license holder to reproduce the material. To view a copy of this license, visit http://creativecommons.org/licenses/by/4.0/ 